\title{
High Performance Mine Transient Electromagnetic System and Applications
}

\author{
Zheng Xiaoliang ${ }^{*}$
}

School of Electric and Information Engineering, Anhui University of Science and Technology, Huainan, Anhui, 232001, P.R. China

\begin{abstract}
This thesis introduces structures and main key technologies of high performance transient electromagnetic system of mine. Such system applies sectional transmitting coil with damping absorption circuit to shorten turn-off time, thus achieving linear falling edge of transmitter current; inspects the turn-off time and current falling edge to enrich essential data of primary field; uses programmable and variable receiving coil to provide different selection modes to solve influence of transient process; and also takes advantages of the pre-amplification and programmable amplification circuit and variable settings of sample period to solve the accurate collection problem for different period signals in secondary field. It provides detection layout and result of application examples proving good test effect and superior system performance.
\end{abstract}

Keywords: Transient electromagnetic, mine, transmitter coil, advanced detection.

\section{INTRODUCTION}

Mine transient electromagnetic method (TEM) is a new developed underground physical detection technology for overcoming the limitation of mine direct current method in underground construction and the difficulty of grounding electrode. With the advantages of convenient construction, small volume effect, large detection range, this method achieved good application effects in the advanced detection of guide aquosity to the interior coalface, as well as the roadway excavation ahead of blind faults, collapse column, hydrological borehole and other constructions [1]. The recent main mine transient electromagnetic instruments products are PROTEM-47 transient electromagnetic system produced by the Canada Geonics company, YCS2000 mine transient electromagnetic instrument developed by China Coal Technology \& Engineering Group Research Institution, YSC40 mine intrinsic safety type transient electromagnetic instrument produced by Chongqing Research Institute of Coal Science Research Institute, TEMHZ75 mine intrinsic safety type transient electromagnetic instrument produced by China University of Geoscience High-tech Resources Detecting Instrument Research Institute, YCS600-I mine intrinsic safety type transient electromagnetic instrument from Gonow Flourish in Shang Xi Geological Instrument Co., LTD and YES40(A) mine transient electromagnetic instrument of Fujian Huahong Intelligent Technology Development Co., LTD, etc.

There is a very big effect on the measurement of waveform quality of TEM transmitter current. The bipolar TEM pulse current waveform with short turn-off time, heavy current and falling edge of high linearity is the focus of current study of TEM lift-off technology [2]. TEM signal features a large dynamic range, the early signal attenuation speed and the weak late signal. Besides, the effect on the early signal from transient process of induced current produced by the receiving coil at the moment when the field disappears can not be ignored. So instrument receiving part should have a high resolution, strong anti-interference ability, high sampling speed and large dynamic range, etc. [3] Designing a high-performance mine transient electromagnetic system with sectional small transmitter coil adding damping absorbing circuit can shorten the turn-off time effectively, can get transmitted waveform with high falling edge and can record turn-off time and the changing process of transmitter current during the turn-off transient. We can eliminate the effects of transient process with the programmable variable receiving coil, and solve the collection problem in a wide range of signals plus the pre-amplification and programmable amplifier circuits, adopting different sample intervals and magnification times for the TEM signals of different periods. The performance of the system has been proved excellent and detection effects of mine transient electromagnetic method have been improved a lot by the validation of application examples.

\section{SYSTEMATIC DESIGN}

The mine transient electromagnetic equipments require to be smaller. The design integrates the transmitting and receiving parts with coils adopting integrated turns small loop, and connects with hardware while transmitting and receiving to share the sync signal and to be free from the GPS synchronization controller used in ground equipment. The overall structure of the system is shown as Fig. (1). The control part is composed by three controllers and the main controller is responsible for the connection with the outside 
world and the control of transmitting and receiving. The other two controllers control the transmitter and receiving part respectively. The transmitter coil adopts sectional multiturn coils of $2 \mathrm{~m} * 2 \mathrm{~m}$. Countdown circuit controls the transmitter current of positive and negative directions from the transmitter coil. Damping absorbing circuit helps the transmitter coil release the energy quickly to make the transmitter current zero rapidly and shorten the turn-off time. transmitter monitoring circuit records the state of transmitter coil, the voltage and current during transmitter and the turnoff time to offer the primary field data for the following data analysis and improve the detecting ability. Receiving coil can eliminate the influence of the transient process with SPC variable type. Preamplifier circuit and programmable amplifier circuit both adopt gain optional way. Analog-todigital conversion (AD) sampling circuit selects the AD chips with high performance. Battery adopts two groups of built-in rechargeable NI-MH batteries to provide current for transmitter coil, forming the primary field.

\section{KEY TECHNOLOGY}

\subsection{Sectional Transmitter Coil}

The transmitter current of TEM is to establish the primary field, and the receiver collects the secondary field signal. While the transmitter coil has small resistance and large inductance values, so the turn-off time can never be zero. In the early period, when there are primary field signals in the secondary ones, the classical way is to abandon this piece of data, which leads to the shallow areas under the surface become the measuring blind zone [4]. The key problems of transmitter are how to realize the short turn-off time and high linearity of falling edge. Domestic scholars deal with the problems by proposing many kinds of constant voltage clamping circuits, such as can feed type constant voltage clamping pulse current, constant voltage pulse lamping circuit with DC voltage regular circuit as the constant voltage source, transmission circuit of constant voltage lamping circuit plus damping absorbing circuit, TVS (transient voltage suppressors) and SIDAC (silicon diode for alternating current) [5-8]. However, these methods don't meet the requirements of the coal mine safety for having complex control circuits and introducing high clamping voltage. We need to design a kind of transmission circuit with sectional transmitter coil plus damping absorbing circuit, whose structure is shown as the Fig. (2). The complete transmitter coil can be divided into two segments L1 and L2 with the switch K5 and there is one absorb damping circuit in charge of the discharge for each segment. K5 closes when transmits, and opens when turn off. Other four switches $\mathrm{K} 1 \sim \mathrm{K} 4$ form an inverter bridge to ensure the positive and negative alternating current go through the transmitter coil.

\subsection{Testing of Turn-off Time and Collection of transmitter Current}

It is very important for the data processing in secondary field to get the accurate measurement of turn-off time and the changing situations collection of transmitter current after turn-off. It will concatenate sampling resistor in the transmitter coil, convert the transmitter current values into voltage values, and send the one voltage to the high precision analog to digital conversion device to collect the falling edge data of the transmitter current after turn-off according to certain sampling periods. The other voltage is sent to be compared by the comparator and the output is as a sign of transmitter current dropping to zero when the controller can be accessed. The specific detection methods are as follows: Start the timer and begin to count when stop transmitting, and when the current deceases to zero, raise the interrupt program and stop counting when the output of comparator changes from high level to low one. Then we can get the turn-off time by calculating the timing time of the timer. This method can get accurate measurement of turn-off time, making it convenient for the later data correction.

\subsection{Variable Programmable Control Receiving Coil}

The working principle of TEM is that the transmitter current sets up a magnetic field in total space and detects the

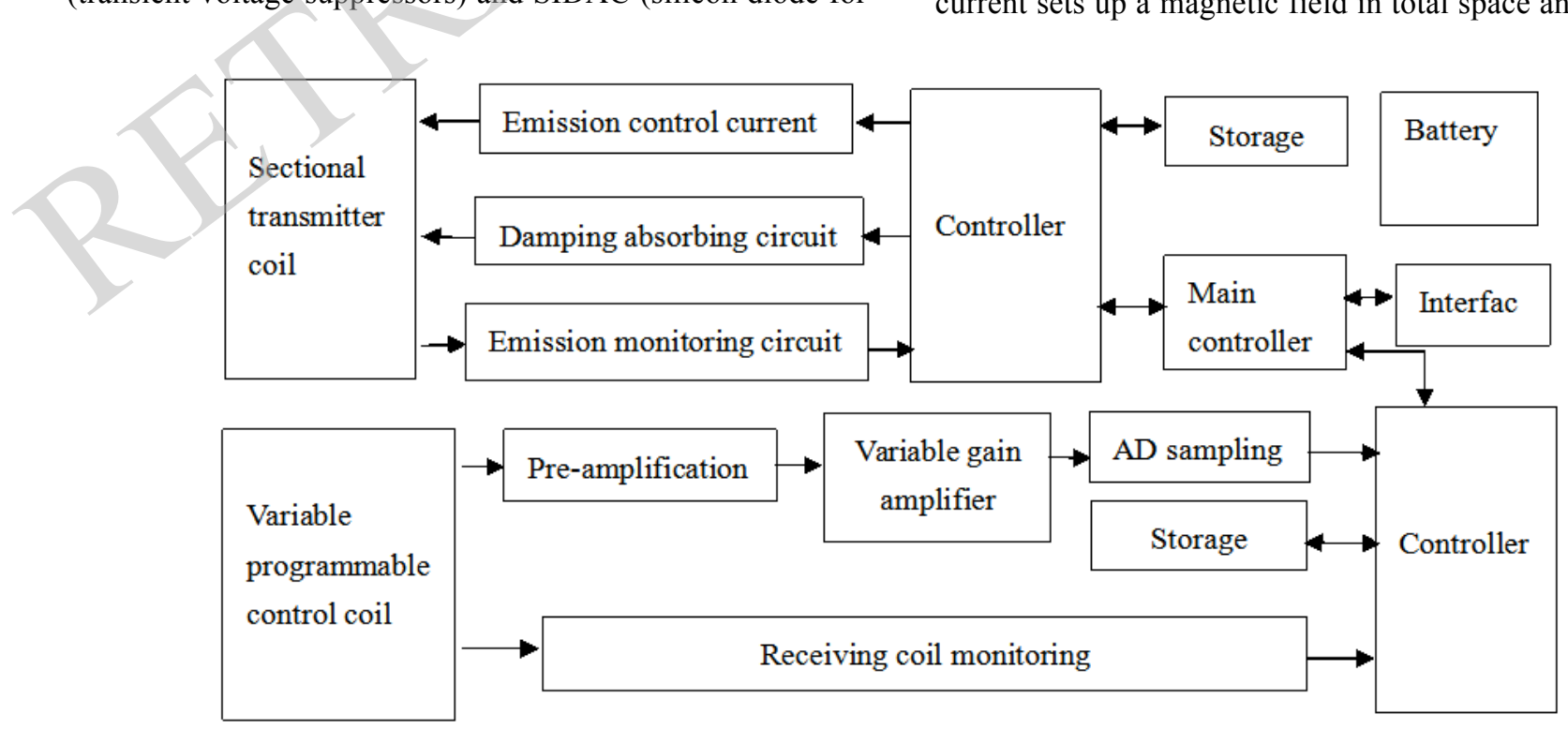

Fig. (1). Structure chart of system. 
produced secondary field signals by receiving coil after turnoff transmitter current. But after this, receiving coil can induct not only the voltage from the secondary field but also the receiving coil induction voltage when the primary field disappears. So the early period signals of TEM mix the transient processes from both secondary and primary field in the receiving coil. The influence of the transient process must be solved to get the pure secondary field signals in the early period. The numerical methods can use turn-off time and closing break falling edge transmitter current data to eliminate the transient process. Also the influence can be eliminated by the hardware structure of programmable receiving coil $[9,10]$.

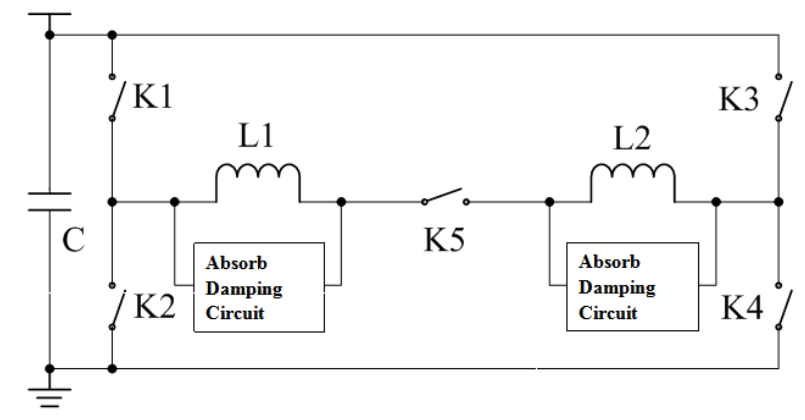

Fig. (2). Structure chart of sectional transmitter coil.

Including the two methods in designing can make the detection data more accurate. The structure of variable programmable control receiving coil is shown as the Fig. (3), in which $\mathrm{A}$ and $\mathrm{B}$ are two coils with identical parameter, $\mathrm{A}+$, $\mathrm{A}-$ and $\mathrm{B}+, \mathrm{B}-$ are two output ends of $\mathrm{A}$ and $\mathrm{B}$ respectively, $\mathrm{KC} 1, \mathrm{KC} 2, \mathrm{KC} 3, \mathrm{KC} 4, \mathrm{KC} 5, \mathrm{KC} 6$ are six switches, 1, 2, 3, 4 are four binding posts used for selection of the coil working mode. There are two working modes, namely non programmable receiving coil method and programmable receiving coil method. The former one is an ordinary coil concatenated by $\mathrm{A}$ and $\mathrm{B}$ which only needs to short connect the 2 and 3 binding posts, switch off $\mathrm{KC} 1, \mathrm{KC} 2, \mathrm{KC} 3, \mathrm{KC} 4$, $\mathrm{KC} 6$, and turn on $\mathrm{KC} 5$. The latter one is the changing connection status by different switch combinations of $\mathrm{A}$ and $\mathrm{B}$ coils during the receiving process, aiming at shortening the transient process of receiving coil.

The specific working principle of programmable receiving coil method is as follows: when turn on $\mathrm{KC} 1, \mathrm{KC} 2$, $\mathrm{KC} 3$, and $\mathrm{KC} 5$, and switch off $\mathrm{KC} 4$ and $\mathrm{KC} 6$, it can be seen that coil $\mathrm{A}$ and $\mathrm{B}$ are reverse connected, and the outputs of coil A and B offset each other, and the total output of the receiving coil is " 0 "; when switch off $\mathrm{KC} 1, \mathrm{KC} 2, \mathrm{KC} 3$, and $\mathrm{KC} 5$, and turn on the $\mathrm{KC} 4$ and $\mathrm{KC} 6$, coil $\mathrm{A}$ and $\mathrm{B}$ are positive connected, overlaying each other, and the total output is the sum of induced voltage of A and B [10].

\subsection{Amplification Circuit and Sampling Period Setting}

Receiving coil is equal to a LR coil in fact. We can only reduce $L$ or increase $R$ to reduce the time of receiving coil transient process according to the transient process of receiving coil. Increasing the resistance, i.e. increasing the number of coil turns, must increase the inductance, so it can only reduce the number and area of coil turns to reduce the

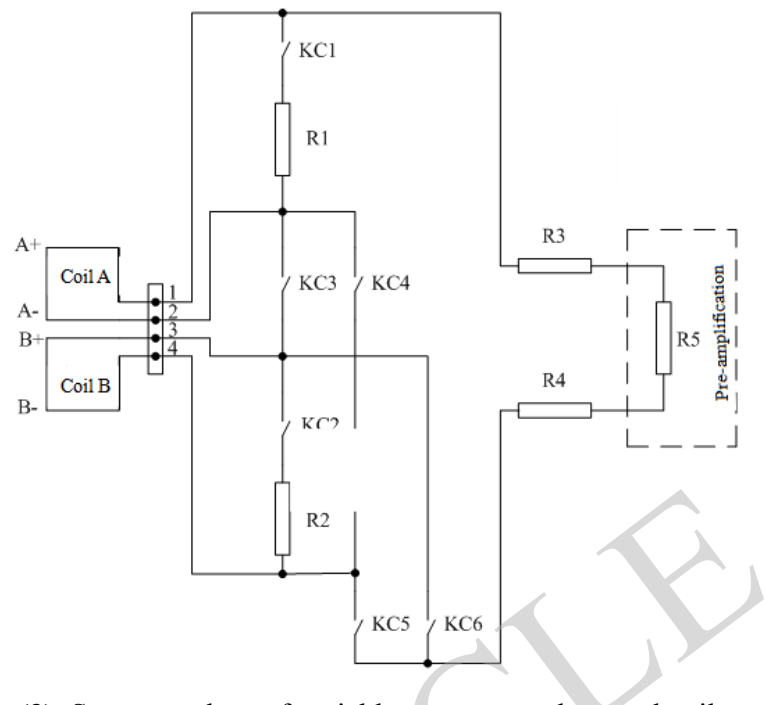

Fig. (3). Structure chart of variable programmed control coil.

inductance L. The receiving coil with reduced turns number and area receives weaker signals, so pre-amplification circuit should be set to primarily amplify the received signals for better detective effect. TEM secondary signal can be divided into early, medium and later stages and signals of different stages have different rates of changing and sizes. The real situation can not be restored by the detected data with same amplification factor and sampling period, so different amplification factors and sampling periods must be set according to the changing characteristics of signals. The best effect can be achieved by adding a variable gain amplification circuit after the pre-amplification circuit to control the amplification factor and setting different sampling periods while programming software according to the characteristics of signals in different periods [11]. Sampling interval and corresponding amplification factors can be divided into three grades according to the characteristics of the secondary field signals changes. Table 1 shows the selection of combination between amplification factor and sampling interval in early, medium and later stage. There are four selections in total. If the first one is selected, the amplification is $0.1,1$ and 10 , the sampling interval $10 \mu \mathrm{s}, 20$ $\mu \mathrm{s}$ and $30 \mu \mathrm{s}$ in early, medium and later stage respectively. And the rest three selections are in the same way. Analog-todigital conversion circuit realize the high precision conversion with the high bandwidth of 24 industrial analogdigital converter ADS1271.

\section{APPLICATION EXAMPLES}

\subsection{Basic Profile}

A mine tries to improve capacity technically, and the 160 yard cross-drift roadways are about to expose the F3 fault. For ensuring the construction safety and with the principle of "detecting before digging, detecting while digging", transient electromagnetic technique is used to advanced detect, providing the basis for eliminating the hidden danger of water.

In the mining area, $3 \sim 5$ coal sandstone fissure aquifer (or water-bearing section) and 6 coal sandstone fissure aquifer 
Table 1. Early, Medium and Later Stage Parameter Selection.

\begin{tabular}{|c|c|c|c|c|c|}
\hline Amplification & Group 1 & Group 2 & Group 3 & Group 4 & Sampling Interval \\
\hline \hline Early stage & 0.1 & 1 & 10 & 100 & $10 \mu \mathrm{s}$ \\
\hline Medium stage & 1 & 10 & 100 & 1000 & $20 \mu \mathrm{s}$ \\
\hline Later stage & 10 & 100 & 1000 & 10000 & $30 \mu \mathrm{s}$ \\
\hline
\end{tabular}

(or water-bearing section) are the direct water filling aquifers. Generally the water abundance is weak for it is controlled by the development degree of tectonic fracture. Quaternary holocene pore aquifer (group) is the indirect water filling aquifers and one of the water supplies in the area. The Taiyuan limestone aquifer and the Ordovician limestone aquifer karst fissures are one of the important hidden dangers of the production safety in exploration area for the characteristics of high water pressure, fierce power, large water quantity and strong water abundance. Hydrogeological condition is medium type. The fault fall of F3 is more than thirty meters, and the zone extending length is $2.3 \mathrm{Km}$, and dripping, spraying and even water bursting may happen during lithology crushing and fracture development.

\subsection{Detecting Arrangements and Requirements}

For the direction of transient electromagnetic detecting coil is sensitive detection one, roadway head-on advanced detection coil is close to the head, normal direction pointing ahead, and the moving distance is usually $0.1 \sim 0.5 \mathrm{~m}$; outside the head-on corner, a certain angel can be adjusted to the head-on side for anterior detection, forming a fans observation system. While the roadway side wall is in transient electromagnetic detection, measuring points are usually arranged at the roadway side wall with the distance between $2 \sim 5 \mathrm{~m}$. The coil normal points into the side or forms an angle with the side. The information of apparent resistivity can be observed point by point $[12,13]$.

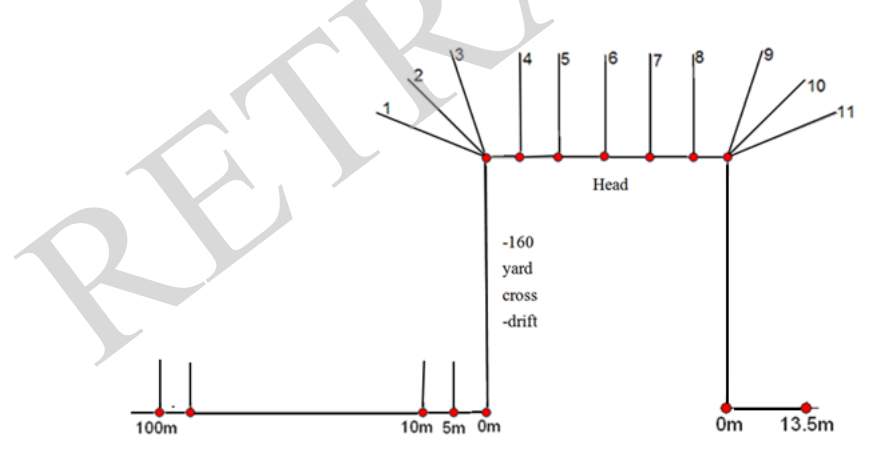

Fig. (4). Schematic plot of points disposition.

The measuring line can be disposed on the south side of Mount Huifengshang and the head of -160 car yard crossdrift. From left to right, the car yard cross-drift head adopts the fans observation system with eleven measuring points and each point detects the directions of the roadway head-on roof, bedding and baseboard. The mountain is arranged along the F3 fault, so the measuring lines in southern side and help detect the fault location outside, arranging the measuring lines to the direction of Mount Huifengshang along the roadway head with the length of about $119 \mathrm{~m}$, measuring points distance of $5 \mathrm{~m}$ and the total number of 24 . Every measuring point detects the horizontal bedding and floor direction respectively. The schematic plot of points disposition as shown as the Fig. (4).

\subsection{Detection Results}

Fig. (5) is the apparent resistivity profile of transient electromagnetic exploration in southern part of Mount Huifengshang, and the total apparent resistivity in detection area is $0 \sim 40 \Omega \cdot \mathrm{m}$, progressive increasing from cool hue to dim hue. On the lateral of the profile, $-13 \sim 20 \mathrm{~m}$ means the relatively higher segment values, and $20 \mathrm{~m} \sim 100 \mathrm{~m}$ means the relatively low resistivity. On the longitudinal, $15 \sim 38 \mathrm{~m}$ means the higher resistivity while in $38 \sim 100 \mathrm{~m}$ the resistivity decreases apparently with the abnormal apparent resistivity gradient. Combining the geological conditions of this area, the gradient abnormality is corresponding to the F3 fault strike location, and the difference in fault hanging wall and footwall apparent resistivity is obvious, resistivity decreasing obviously in the fault zone.

Fig. (6) is the profile of advanced detective apparent resistivity section of -160 yard's head-on cross-drift, with the direction of roof, bedding and baseboard. The apparent resistivity values are relatively high overall in each profile, and the characteristic is the same as the high resistance area in the detection result of southern Mount Huifengshang. It can be seen from the comparison, there are local relatively low resistivity zones (less than $30 \Omega \cdot \mathrm{m}$ ) which explained as YC1 and YC2 in the front of the head with $30 \mathrm{~m}$ and $75 \mathrm{~m}$ respectively.

According to comprehensive inference, the aquosity is relatively large in the fault $\mathrm{F} 3$, while it is uneven in the fault plane direction because of the differences in the geological factors such as mine supply conditions, development degree of fault rock fissure, filling and cementation degree. Though -160 yard cross-drift is in the high resistance area, and the aquosity is less, there are still some relatively low resistance areas, and plus the driftage effects as well as adding new fissure water channels, so the hydrological anomalies such as dripping and spraying may happen when the driftage is through the fault.

\section{CONCLUSION}

We get the linear current falling age with the new transmitter receiving coil by reducing transmitter turn-off 
The apparent resistivity profile of transient electromagnetic on the return air south for bedding

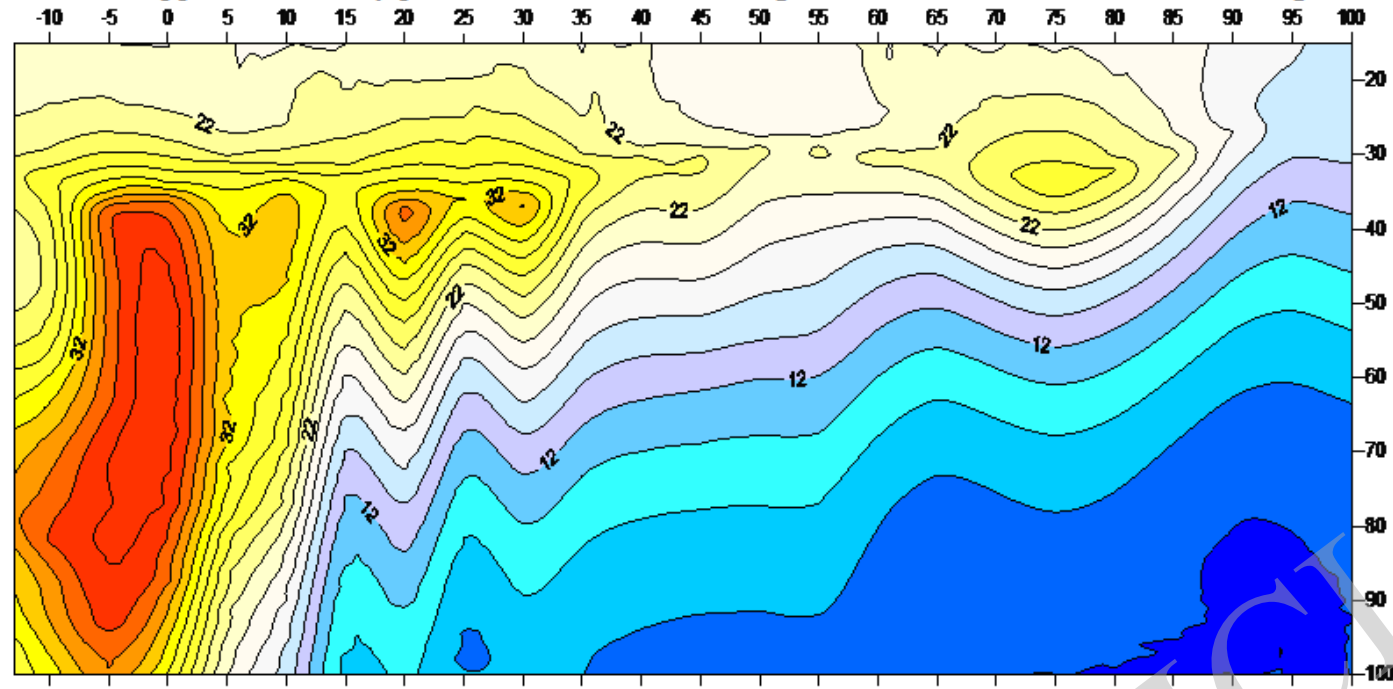

The apparent resistivity profile of transient electromagnetic on the return air south for bottom

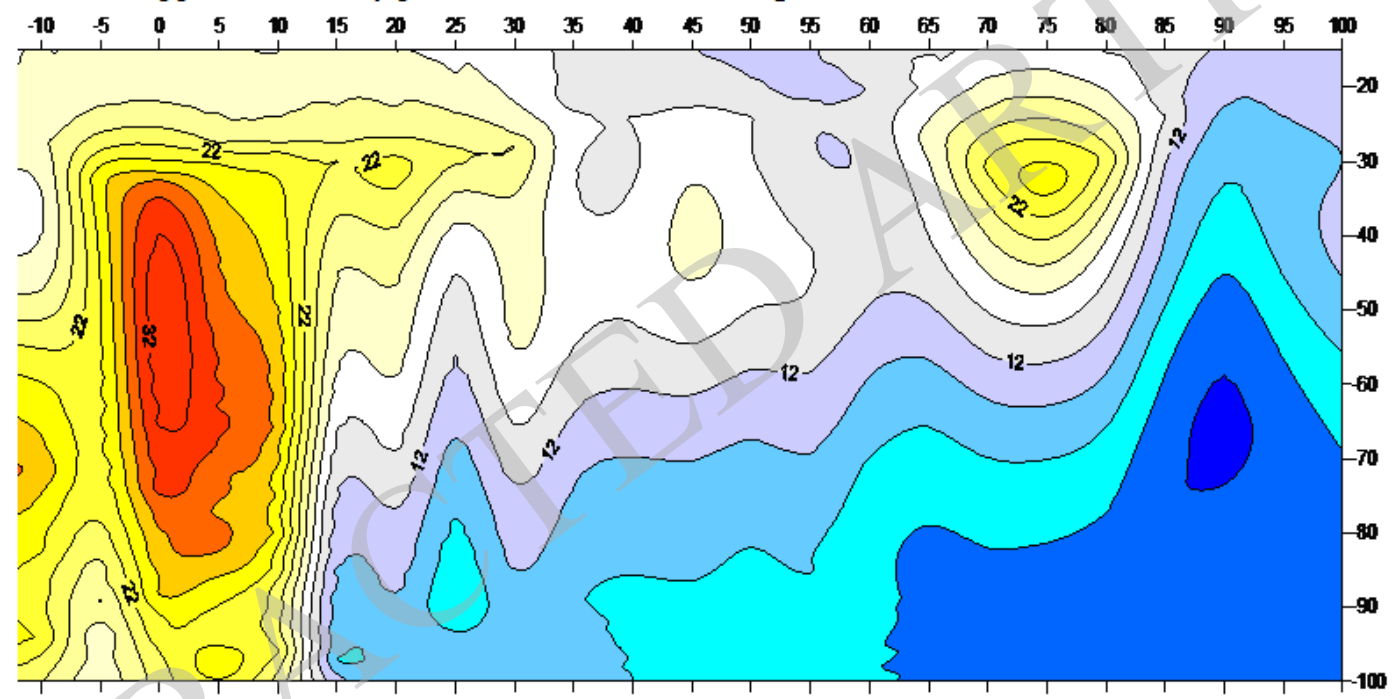

Fig. (5). Apparent resistivity section of south air rise roadway.
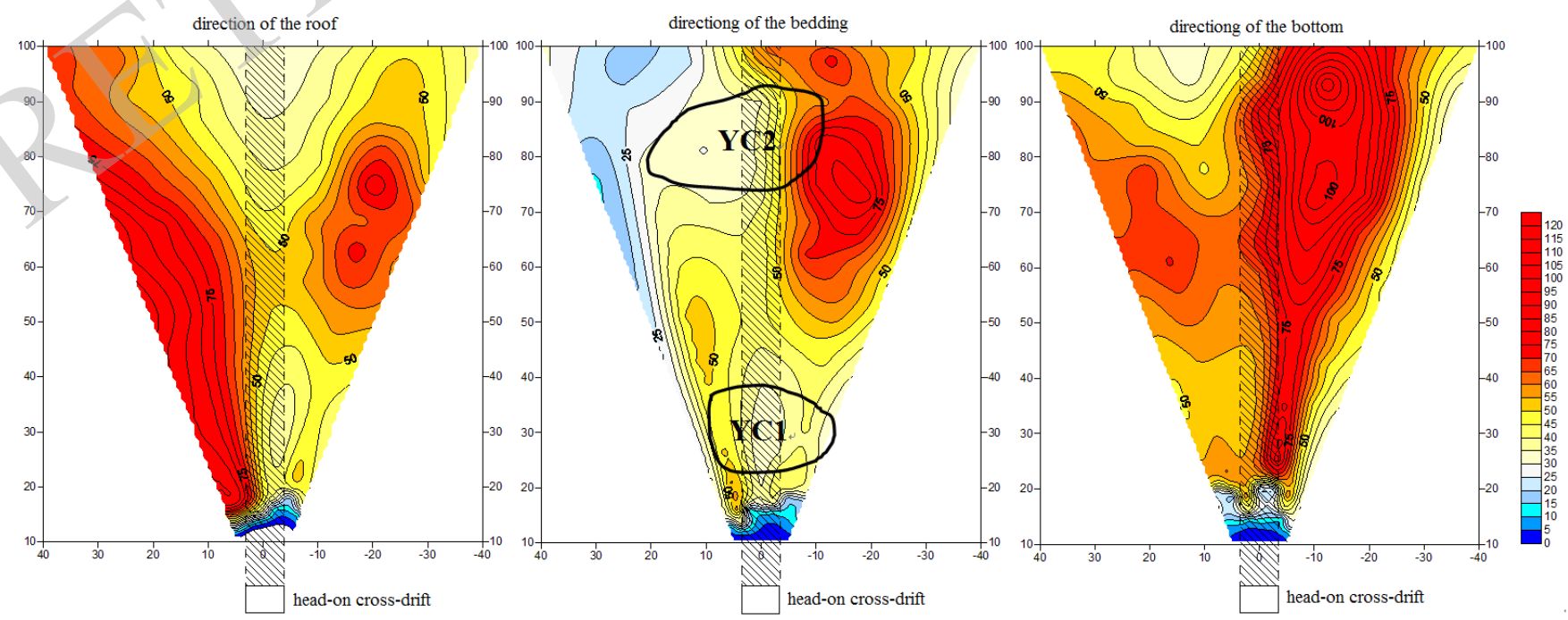

Fig. (6). Apparent resistivity section of -160 yard's head-on cross-drift. 
time effectively and grasp the the turn-off time and the change of current accurately with recording the transmitter current falling edge which offer the primary field data for late data processing. Variable programmed control receiving coil offers two receiving ways to eliminate the influence of transient process effectively. Designing the pre-amplification and programmed control amplification circuit with alternative amplification times and cooperating with different sampling periods can meet the detection demand of the changing signals in different stages of secondary field to record the change of secondary field more accurately. Choosing analog-digital converter of 24 precision can improve the conversion accuracy, and software programming can filter out interference from $50 \mathrm{~Hz}$ frequency signals with the method of the adaptive smoothing. The application results show that the developed high-performance mine transient electromagnetic system has good performance.

\section{CONFLICT OF INTEREST}

The author confirms that this article content has no conflict of interest.

\section{ACKNOWLEDGEMENTS}

Declared none.

\section{REFERENCES}

[1] Jiang, Z.; Yue, J.; Liu, S. Mine transient electromagnetic

\section{observation system of smallmulti-turn coincident configuration. $J$. China Coal Soc., 2007, 32, 1152-1156.}

[2] Fu, Z.; Zhou, L.; Su, X. Current impulse rectifying technique for transient electromagnetic transmitter. Power Electron., 2006, 40, 108-111.

[3] Li, S.; Li, C.; Zhang, Y.; Zhang, P.; Song, J. Study of the key technology in TEM instrument. Coal Geol. Explor., 2011, 29, 5558 .

[4] Wang, J.; Lin, J. Realization of high speed data collecting system in ATEM. J. Jilin Univ. (Inform. Sci. Ed.), 2004, 22, 537-540.

[5] Fu, Z.; Zhao, J.; Zhou, L.; Luo, Q.; Su, X. WTEM fast turnoff transient electromagnetic detection system. Chin. J. Scientific Instrum., 2008, 29, 933-936.

[6] Du, M.; Fu, Z.; Zhou, L. Research on energy-feedback and constant voltage clamp bipolar pulse current source. Trans. China Electrotech. Soci., 2007, 22, 57-62.

[7] Zhao, H.; Liu, L.; Wu, K.; Zhang, J.; Fang, G. Voltage stabilized clamping and fast turn-off transient electromagnetic transmitting system. Chin. J. Sci. Instrum., 2013, 34, 803-808.

[8] Fu, Y.; Yang, Y.; Fu, Z.; Su, X. Passive constant pressure clamping technology for transient electromagnetic transmitter. Transd. Microsys. Technol., 2011, 30, 46-49.

[9] Li, S.; Li, C.; Zhang, Y.; Zhang, P.; Song, J. Study of the key technology in TEM instrument. Coal Geol. Explor., 2011, 29, 5558

[10] Li, S.; Li, C.; Zhang, P.; Song, J.; Zhao, C. A New program controlled antenna to measure early signal in TEM. Chin. J. Sci.. Instrum., 2002, 23, 192-194.

[11] Li, C.; Li, S.; Song, J.; Tang, T. Study on detection method of early signal in TEM. Coal Geol. Explor., 1999, 27, 58-60.

[12] Wang, D.; Liu, Z.; Wu, J.; Xu, Y. Application of mine transient electromagnetic method to advanced detecting of roadway head. Chin. J. Eng. Geophy., 2011, 8, 403-407.

[13] Zhang, J.; Zhao Y.; Li, P. Application research of mine transient electromagnetic method in advanced detection. Chin. J. Eng. Geophy., 2012, 9, 49-53. 

Received: November 26, 2014

This is an open access article licensed under the terms of the Creative Commons Attribution Non-Commercial License (http://creativecommons.org/licenses/by-nc/3.0/) which permits unrestricted, non-commercial use, distribution and reproduction in any medium, provided the work is properly cited. 